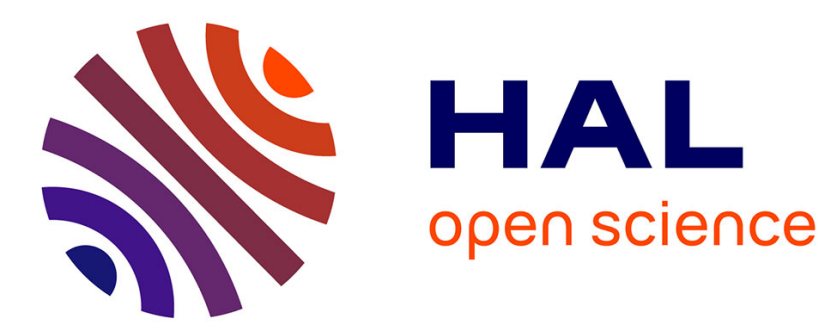

\title{
An inverse spectral problem for a schrodinger operator with unbounded potential
}

Laure Cardoulis, Michel Cristofol, Patricia Gaitan

\section{To cite this version:}

Laure Cardoulis, Michel Cristofol, Patricia Gaitan. An inverse spectral problem for a schrodinger operator with unbounded potential. Inverse Problems, 2003, 19, pp.467-476. hal-00003752

\section{HAL Id: hal-00003752 \\ https://hal.science/hal-00003752}

Submitted on 3 Jan 2005

HAL is a multi-disciplinary open access archive for the deposit and dissemination of scientific research documents, whether they are published or not. The documents may come from teaching and research institutions in France or abroad, or from public or private research centers.
L'archive ouverte pluridisciplinaire HAL, est destinée au dépôt et à la diffusion de documents scientifiques de niveau recherche, publiés ou non, émanant des établissements d'enseignement et de recherche français ou étrangers, des laboratoires publics ou privés. 


\title{
An Inverse Spectral Problem for a Schrödinger Operator with an Unbounded Potential.
}

\author{
Laure Cardoulis Michel Cristofol Patricia Gaitan* \\ January 3, 2005
}

*Université de Toulouse 1, Place Anatole France, 31000 Toulouse, cardouli@math.univ-tlse1.fr;Université de Provence, CMI,UMR CNRS 6632, 39, rue Joliot Curie, 13453 Marseille Cedex 13, France, Université d'Aix Marseille III, IUT de Saint Jerôme, cristo@cmi.univ-mrs.fr; Université de Provence, CMI,UMR CNRS 6632, 39, rue Joliot Curie,13453 Marseille Cedex 13, France, Université de la Méditerranée, IUT d'Aix en Provence. 


\begin{abstract}
In this paper, we prove a uniqueness theorem for the potential $V(x)$ of the following Schrödinger operator $H=-\Delta+q(|x|)+V(x)$ in $\mathbb{R}^{2}$, where $q(|x|)$ is a known increasing radial potential satisfying $\lim _{|x| \rightarrow+\infty} q(|x|)=+\infty$ and $V(x)$ is a bounded potential.
\end{abstract}

\title{
1 Introduction
}

Recently, a lot of papers have dealt with inverse problems for Schrödinger Operators in the whole space or in half space (see for example [ER], [GR], [I1], [W]). In all these papers, the potential has to be decreasing towards infinity.

Our aim is to study an inverse problem for a potential which tends to infinity at infinity. There are many papers on inverse spectral problems for increasing potentials on the line or on the half-line (see [GS1], [GS2]) but to our knowledge, there are no results in $\mathbb{R}^{2}$.

Let $Q$ be a potential such that:

$$
\begin{gathered}
Q \in L_{l o c}^{1}\left(\mathbb{R}^{2}\right), \\
Q(x) \geq \alpha \text { where } \alpha \text { is a constant, } \\
\lim _{|x| \rightarrow+\infty} Q(x)=+\infty .
\end{gathered}
$$

We recall the well-known result that the operator $H=-\Delta+Q$, considered as an operator in $L^{2}\left(\mathbb{R}^{2}\right)$, admits a compact inverse. Its spectrum is discrete and consists of eigenvalues: $\lambda_{1} \leq \lambda_{2} \leq \ldots \leq \lambda_{n} \rightarrow_{n \rightarrow+\infty}+\infty$. Furthermore, the first eigenvalue $\lambda_{1}$ is simple and associated with an eigenfunction $\phi_{1}>0$. (see [Ab], [Ag1], [Ag2], [EE]). In the present paper, we shall consider the following problem

$$
(-\Delta+q(|x|)+V(x)) u=\lambda u \text { in } \mathbb{R}^{2}
$$

where $\lambda \in \mathbb{R}^{*+}, q$ is a known increasing radial potential satisfying $q(|x|) \in C^{2}(\mathbb{R})$;

$\lim _{|x| \rightarrow+\infty} q(|x|)=+\infty$ and $V$ is a bounded potential.

Denote $|x|=r$ and

$$
C_{\lambda, p}(V):=\lim _{r \rightarrow+\infty} 2 q^{1 / 4} r^{1 / 2} e^{\int\left(q^{1 / 2}-\frac{\lambda}{2} q^{-1 / 2}\right)} \int_{0}^{2 \pi} u(r, \theta) e^{-i p \theta} d \theta
$$

where $\int\left(q^{1 / 2}-\frac{\lambda_{l}}{2} q^{-1 / 2}\right)$ denotes a primitive of $\left(q^{1 / 2}-\frac{\lambda_{l}}{2} q^{-1 / 2}\right)$ and $u$ is a solution of (1.1). Consider the following statements:

(h1) $q(|x|)+V(x) \geq \alpha$ where $\alpha$ is a constant

(h2) $\forall N \in \mathbb{N}^{*}, \exists C_{N} \in \mathbb{R}^{*+},|V(r, \theta)| \leq C_{N}(1+r)^{-N} e^{-\int q^{1 / 2}}$

(h3) $\lim _{r \rightarrow+\infty} \frac{q^{\prime}(r)}{q^{3 / 2}(r)}=0$

(h4) $\frac{q^{\prime \prime}(r)}{q^{3 / 2}(r)}=O\left(\frac{1}{r^{2} q^{1 / 2}(r)}, \frac{1}{q^{3 / 2}(r)}, \frac{q^{\prime}(r)}{q^{2}(r)}\right)$, and $\frac{q^{\prime 2}(r)}{q^{5 / 2}(r)}=O\left(\frac{1}{r^{2} q^{1 / 2}(r)}, \frac{1}{q^{3 / 2}(r)}, \frac{q^{\prime}(r)}{q^{2}(r)}\right)$

(h5) $\frac{1}{r^{2} q^{1 / 2}(r)}, \frac{1}{q^{3 / 2}(r)}$ and $\frac{q^{\prime}(r)}{q^{2}(r)} \in L^{1}[1,+\infty[$ 
(h6) $q^{-1 / 4} e^{-\int\left(q^{1 / 2}-\frac{\lambda}{2} q^{-1 / 2}\right)} \in L^{2}[1,+\infty[$

(h7) $\int_{r}^{+\infty} o\left(e^{-2 \int\left(q^{1 / 2}-\frac{\lambda}{2} q^{-1 / 2}\right)}\right)=o\left(e^{-2 \int\left(q^{1 / 2}-\frac{\lambda}{2} q^{-1 / 2}\right)}\right)$

(h8) $q^{-1 / 4} e^{\int\left(q^{1 / 2}-\frac{\lambda}{2} q^{-1 / 2}\right)} \notin L^{2}[1,+\infty[$

For example, $q(|x|)=|x|^{2}$ satisfies each of the previous hypotheses.

Our aim is to prove the following theorem.

\section{Theorem:}

Let $V(x)$ and $W(x)$ be two bounded potentials with compact support, both satisfying $(h 1)$ and $(h 2)$. Denote by $\left(\lambda_{l}(V)\right)_{l}$ (resp. $\left.\left(\lambda_{l}(W)\right)_{l}\right)$ the eigenvalues of the operator $-\Delta+q(|x|)+V(x)$ (resp. $-\Delta+q(|x|)+W(x))$. Assume also the validity of $(h 3)$ to $(h 8)$.

$$
\text { If } \forall l \in \mathbb{N}^{*}, \forall p \in \mathbb{N},\left\{\begin{array}{l}
\lambda_{l}(V)=\lambda_{l}(W) \\
\text { and } \\
C_{\lambda_{l}, p}(V)=C_{\lambda_{l}, p}(W)
\end{array} \text { then } V=W\right. \text {. }
$$

We shall follow a method used by T.Suzuki in a bounded domain [Su]. This method has been generalized by H.Isozaki [I2] for the anharmonic operator in $\mathbb{R}^{2}$. In the first step, we study the asymptotic behaviour of the solutions of a second order differential equation which stems from equation (1.1). Then we prove that under hypotheses (h1) to (h8), the constant $C_{\lambda_{l}, p}(V)$ exists. In the second step, we prove that the first eigenfunctions associated with $V$ and $W$ are the same, and since the first one is strictly positive, we are able to come to the above conclusion.

Remarks:

Note that $C_{\lambda_{l}, p}$ is a theoretical constant whose physical interpretation is not easy.

The hypothesis $C_{\lambda_{l}, p}(V)=C_{\lambda_{l}, p}(W)$ is rather weak, because we need not take care of the multiplicity of the eigenvalues nor of any normalization of the eigenfunctions.

\section{Asymptotic behaviour of a solution}

Here we study the asymptotic behaviour of the solutions of the following equation :

$$
(-\Delta+q(|x|)+V(x)) u=\lambda u \text { in } \mathbb{R}^{2}
$$

where $q$ is a known increasing radial potential satisfying $q(|x|) \in C^{2}(\mathbb{R})$;

$\lim _{|x| \rightarrow+\infty} q(|x|)=+\infty$ and $V$ is a bounded potential.

Put $|x|=r$. We use polar coordinates to define $u_{p}$

$$
u_{p}(r)=r^{1 / 2} \int_{0}^{2 \pi} u(r, \theta) e^{-i p \theta} d \theta .
$$

Using (1.1) and (2.1), we get :

$$
-u_{p}^{\prime \prime}(r)+\left(\frac{p^{2}-1 / 4}{r^{2}}+q(r)-\lambda\right) u_{p}(r)=f_{p}(r),
$$


where

$$
f_{p}(r)=-r^{1 / 2} \int_{0}^{2 \pi} V(r, \theta) u(r, \theta) e^{-i p \theta} d \theta .
$$

We are now able to prove the following theorem.

Theorem 2.1 Let $q$ and $V$ be potentials defined as before, satisfying (h1) to (h8), $\lambda$ and a two positive reals. Then the equation

$$
-u^{\prime \prime}(r)+\left(\frac{a}{r^{2}}+q(r)-\lambda\right) u(r)=0
$$

has a system of fundamental solutions with the asymptotic behaviour:

$$
\begin{gathered}
\overline{\bar{u}}_{1} \sim \frac{1}{2} q^{-1 / 4} e^{-\int\left(q^{1 / 2}-\frac{\lambda}{2} q^{-1 / 2}\right)} \text { for } r \rightarrow+\infty, \\
\overline{\bar{u}}_{2} \sim q^{-1 / 4} e^{\int\left(q^{1 / 2}-\frac{\lambda}{2} q^{-1 / 2}\right)} \text { for } r \rightarrow+\infty .
\end{gathered}
$$

Furthermore, the Wronskian $\overline{\bar{u}}_{1} \overline{\bar{u}}_{2}^{\prime}-\overline{\bar{u}}_{1}^{\prime} \overline{\bar{u}}_{2}=1$.

\section{Proof :}

\section{First step}

We set $y=\left(\begin{array}{c}u \\ u^{\prime}\end{array}\right)$; using (2.4) we get :

$$
y^{\prime}=\left(\begin{array}{c}
u^{\prime} \\
u^{\prime \prime}
\end{array}\right)=\left(\begin{array}{cc}
0 & 1 \\
q(r)-\lambda+\frac{a}{r^{2}} & 0
\end{array}\right)\left(\begin{array}{c}
u \\
u^{\prime}
\end{array}\right)=\left(\begin{array}{cc}
0 & 1 \\
q(r)-\lambda+\frac{a}{r^{2}} & 0
\end{array}\right) y .
$$

We denote

$$
k=k(r)=\sqrt{q(r)-\lambda+\frac{a}{r^{2}}}, \quad P=\left(\begin{array}{cc}
1 & -1 \\
k & k
\end{array}\right), \quad y=P \widetilde{y} .
$$

If we combine the two relations (2.7) and (2.8), we obtain :

$$
\widetilde{y}^{\prime}=P_{1} \widetilde{y} \text { with } P_{1}=P^{-1}\left[\left(\begin{array}{cc}
k & k \\
k^{2} & -k^{2}
\end{array}\right)-P^{\prime}\right],
$$

where $P^{\prime}$ is the derivative of the matrix $\mathrm{P}$ with respect to the variable $r$.

Next, we compute for each term of the matrix $P_{1}$, an appropriate Taylor polynomial; this allows us to write (2.9) in the form

$$
\widetilde{y}^{\prime}=\left(\begin{array}{cc}
q^{1 / 2}-\frac{\lambda}{2 q^{1 / 2}}-\frac{q^{\prime}}{4 q} & -\frac{q^{\prime}}{4 q} \\
-\frac{q^{\prime}}{4 q} & -q^{1 / 2}+\frac{\lambda}{2 q^{1 / 2}}-\frac{q^{\prime}}{4 q}
\end{array}\right) \widetilde{y}+R\left(\frac{1}{r^{2} q^{1 / 2}(r)}, \frac{1}{q^{3 / 2}(r)}, \frac{q^{\prime}(r)}{q^{2}(r)}\right) \widetilde{y} .
$$

Remark : $R\left(\frac{1}{r^{2} q^{1 / 2}(r)}, \frac{1}{q^{3 / 2}(r)}, \frac{q^{\prime}(r)}{q^{2}(r)}\right)$ shall be a $2 \times 2$-matrix where all the coefficients are in the form $O\left(\frac{1}{r^{2} q^{1 / 2}(r)}, \frac{1}{q^{3 / 2}(r)}, \frac{q^{\prime}(r)}{q^{2}(r)}\right)=O\left(\frac{1}{r^{2} q^{1 / 2}(r)}\right)+O\left(\frac{1}{q^{3 / 2}(r)}\right)+O\left(\frac{q^{\prime}(r)}{q^{2}(r)}\right)$. 


\section{Second step}

Consider

$$
\widetilde{y}=\left(I-\frac{q^{\prime}}{4 q^{3 / 2}} P_{2}\right) z
$$

We look for $P_{2}$ such that $z^{\prime}=A(q) z+R\left(\frac{1}{r^{2} q^{1 / 2}(r)}, \frac{1}{q^{3 / 2}(r)}, \frac{q^{\prime}(r)}{q^{2}(r)}\right) z$, with $A(q)$ a diagonal matrix. Then (2.10) can be written :

$$
\left.\widetilde{y}^{\prime}=\left[\left(q^{1 / 2}-\frac{\lambda}{2 q^{1 / 2}}\right) A_{0}+\frac{q^{\prime}}{4 q} A_{2}\right] \widetilde{y}+R\left(\frac{1}{r^{2} q^{1 / 2}(r)}, \frac{1}{q^{3 / 2}(r)}, \frac{q^{\prime}(r)}{q^{2}(r)}\right)\right) \widetilde{y},
$$

with $A_{0}=\left(\begin{array}{cc}1 & 0 \\ 0 & -1\end{array}\right)$ and $A_{2}=\left(\begin{array}{cc}-1 & -1 \\ -1 & -1\end{array}\right)$. Substituting the derivative of (2.11) in the equation (2.12) yields

$z^{\prime}=\left[\left(q^{1 / 2}-\frac{\lambda}{2 q^{1 / 2}}\right) A_{0}+\frac{q^{\prime}}{4 q}\left(P_{2} A_{0}-A_{0} P_{2}+A_{2}\right)\right] z+R\left(\frac{1}{r^{2} q^{1 / 2}(r)}, \frac{1}{q^{3 / 2}(r)}, \frac{q^{\prime}(r)}{q^{2}(r)}\right) z$.

We set

$$
A(q)=\left(q^{1 / 2}-\frac{\lambda}{2 q^{1 / 2}}\right) A_{0}+\frac{q^{\prime}}{4 q}\left(P_{2} A_{0}-A_{0} P_{2}+A_{2}\right) .
$$

Then, putting $P_{2}=\left(\begin{array}{cc}0 & -1 / 2 \\ 1 / 2 & 0\end{array}\right)$, we get :

$$
z^{\prime}=A(q) z+R\left(\frac{1}{r^{2} q^{1 / 2}(r)}, \frac{1}{q^{3 / 2}(r)}, \frac{q^{\prime}(r)}{q^{2}(r)}\right) z,
$$

with

$$
A(q)=\left(\begin{array}{cc}
q^{1 / 2}-\frac{\lambda}{2 q^{1 / 2}}-\frac{q^{\prime}}{4 q} & 0 \\
0 & -q^{1 / 2}+\frac{\lambda}{2 q^{1 / 2}}-\frac{q^{\prime}}{4 q}
\end{array}\right) .
$$

\section{Third step}

$\overline{\text { We will look }}$ at the asymptotic behaviour of $z$. We will search a diagonal matrix $E(q)$ such that $E^{\prime}(q)=A(q) E(q)$. Introduce the new variable $v$ defined by $z=E(q) v$, with

$$
E(q)=\left(\begin{array}{cc}
q^{-1 / 4} e^{\int\left(q^{1 / 2}-\frac{\lambda}{2 q^{1 / 2}}\right)} & 0 \\
0 & \frac{1}{2}\left(q^{-1 / 4} e^{-\int\left(q^{1 / 2}-\frac{\lambda}{2 q^{1 / 2}}\right)}\right)
\end{array}\right)=\left(\begin{array}{cc}
E_{1} & 0 \\
0 & E_{2}
\end{array}\right) .
$$

Then we have

$$
v^{\prime}=E^{-1}(q) R(q) E(q) v=\left(\begin{array}{c}
K_{1} \\
K_{2}
\end{array}\right) v .
$$

We define the mapping $T$ and the subset $\mathcal{F}$ as follows :

$$
v \rightarrow\left\{\begin{array}{l}
-\int_{r}^{\infty} K_{1} v(t) d t \\
\xi_{2}+\int_{r_{0}}^{r} K_{2} v(t) d t,
\end{array}\right.
$$


$\mathcal{F}=\left\{v=\left(v_{1}, v_{2}\right) ; \forall i \quad v_{i}:\left[r_{0}, \infty\right) \rightarrow \mathbb{R}, \quad v_{1}=o\left(e^{-2 \int\left(q^{1 / 2}-\frac{\lambda}{2 q^{1 / 2}}\right)}\right), \sup _{r \geq r_{0}}\left|v_{2}(x)\right|<+\infty\right\}$.

Then for a suitable choice of $r_{0}$ and $\xi_{2} \neq 0$, the mapping $T: \mathcal{F} \rightarrow \mathcal{F}$ is a contraction (cf Appendix A), and thus we can find an unique $v$, solution of (2.17). So we get $z$, and thus $\widetilde{y}$, and finally $y$. From the asymptotic behaviour of $z$, which is given via the components of the matrix $E(q)$, we obtain the asymptotic behaviour of $u$ in the form $u \sim E_{1} v_{1}-E_{2} v_{2}$. Since $v \in \mathcal{F}, \frac{E_{1}}{E_{2}} v_{1}=o(1)$ and $v$ tends to $\xi_{2} \neq 0$ as $r$ tends to infinity, we deduce that $E_{2}$ describes an asymptotic behaviour of a solution of (2.4). So we can prove that $E_{1}$ is another asymptotic behaviour of a solution of (2.4), using a standard transformation. Finally, we deduce (2.5) and (2.6), and find that the Wronskian is equal to 1 . This concludes the proof.

First we have to prove that the constant defined by (1.2) actually exists. This is the following lemma :

Lemma 2.1 Let $u$ be in $L^{2}\left(\mathbb{R}^{2}\right)$ such that $(H-\lambda) u=0$ for $\lambda$ a positive real with

$$
H=-\Delta+q(|x|)+V(x),
$$

where $q$ is an increasing radial potential which tends to infinity at infinity and $q$ and $V$ satisfy the hypotheses (h1) to (h8). Then the following limit exists

$$
\lim _{r \rightarrow+\infty} 2 q^{1 / 4} r^{1 / 2} e^{\int\left(q^{1 / 2}-\frac{\lambda}{2} q^{-1 / 2}\right)} \int_{0}^{2 \pi} u(r, \theta) e^{-i p \theta} d \theta=C_{\lambda, p}(V) .
$$

Proof :

Let $u$ be the solution of $(-\Delta+q(|x|)+V(x)) u=\lambda u$. Setting $u_{p}(r)=r^{1 / 2} \int_{0}^{2 \pi} u(r, \theta) e^{-i p \theta} d \theta$, then $u_{p}$ is the solution of the equation

$$
-u_{p}^{\prime \prime}(r)+\left(\frac{p^{2}-1 / 4}{r^{2}}+q(r)-\lambda\right) u_{p}(r)=f_{p}(r),
$$

with $f_{p}(r)=-r^{1 / 2} \int_{0}^{2 \pi} V(r, \theta) u(r, \theta) e^{-i p \theta} d \theta$. Then, by using the theorem 2.1 and the hypotheses (h3) to (h8), we obtain the existence of the limit (2.8) (see Appendix B).

\section{Unicity Theorem}

We can now state the unicity theorem. We consider the operators $-\triangle+q(|x|)+V(x)$ and $-\triangle+q(|x|)+W(x)$, where $q(|x|)$ is an increasing radial potential verifying the hypotheses (h3) to $(h 8), V(x)$ and $W(x)$ having compact support. 
Theorem 3.1 Let $V$ and $W$ be two bounded potentials with compact support. If $\forall l \in \mathbb{N}^{*}, \forall p \in \mathbb{N}$,

$$
\left\{\begin{array}{l}
\lambda_{l}(V)=\lambda_{l}(W) \\
\text { and } \\
C_{\lambda_{l}, p}(V)=C_{\lambda_{l}, p}(W)
\end{array} \quad \text { then } V=W\right.
$$

\section{Proof:}

Let $\lambda_{1}<\lambda_{2}<\ldots$ be the eigenvalues of the operators $-\triangle+q(|x|)+V(x)$ and $-\triangle+q(|x|)+W(x)$.

Let $\varphi_{1}, \varphi_{2}, \varphi_{3}, \ldots$ be the normalized eigenfunctions associated with the potential $V$. Let $\psi_{1}, \psi_{2}, \psi_{3}, \ldots$ be the normalized eigenfunctions associated with the potential $W$. We assume that supp $V \subset\{x ;|x|<R\}$ and suppW $\subset\{x ;|x|<R\}, R$ being a fixed real. We are going to prove that $\varphi_{1}(x)=\psi_{1}(x)$, for all $x$.

\section{First step}

We prove that

$$
\forall l, \varphi_{l}(x)=\psi_{l}(x), \text { if }|x|>R .
$$

Recall that if $|x|>R$ then

$$
\left\{\begin{array}{l}
(-\triangle+q(|x|)) \varphi_{l}=\lambda_{l} \varphi_{l} \\
\text { and } \\
(-\triangle+q(|x|)) \psi_{l}=\lambda_{l} \psi_{l}
\end{array}\right.
$$

We decompose $\varphi_{l}$ and $\psi_{l}$ relative to the trigonometric functions basis $\left\{e^{-i k \theta}\right\}_{k}$, and we prove that all the coefficients are equal. For this purpose, we put

$$
a_{l, k}(r)=\int_{0}^{2 \pi} \varphi_{l}(r, \theta) e^{-i k \theta} d \theta \text { and } b_{l, k}(r)=r^{\frac{1}{2}} a_{l, k}(r) .
$$

If $r>R$ then the function $b_{l, k}(r)$ is the solution of

$$
-b^{\prime \prime, k}(r)+\left(\frac{k^{2}-\frac{1}{4}}{r^{2}}+q(r)-\lambda_{l}\right) b_{l, k}(r)=0
$$

This equation has the following fundamental system of solutions (cf theorem 2.1)

$$
b_{l, k}(r)=C_{1} u_{1}(r)+C_{2} u_{2}(r) \text { with }\left\{\begin{array}{l}
\overline{\bar{u}}_{1} \sim \frac{1}{2} q^{\frac{-1}{4}} e^{\int\left(-q^{\frac{1}{2}}+\frac{\lambda}{2 q^{\frac{1}{2}}}\right)} \text { for } r \rightarrow+\infty \\
\overline{\bar{u}}_{2} \sim q^{\frac{-1}{4}} e^{\int\left(q^{\frac{1}{2}}-\frac{\lambda}{2 q^{\frac{1}{2}}}\right)} \text { for } r \rightarrow+\infty .
\end{array}\right.
$$

But, $b_{l, k}(r) \in L^{2} \Rightarrow C_{2}=0$, so we get that $b_{l, k}(r)=C_{1} u_{1}(r)$.

In the same way, we prove that if $\tilde{b}_{l, k}$ are the functions associated with $\psi_{l}(r)$, then $\tilde{b}_{l, k}(r)=\tilde{C}_{1} u_{1}(r)$. Using the hypotheses

$$
\left\{\begin{array}{l}
\lambda_{l}(V)=\lambda_{l}(W) \\
\text { and } \\
C_{\lambda_{l}, p}(V)=C_{\lambda_{l}, p}(W)
\end{array}\right.
$$


we have

$$
b_{l, k}(r)=\tilde{b}_{l, k}(r), \quad \forall l \text { and } \forall k
$$

This yields the first step.

\section{Second step}

It is necessary to prove that $\varphi_{1}$ and $\psi_{1}$ are equal everywhere. For this, we use an idea of $[\mathrm{Su}]$ who uses this method to solve an interior problem. Note that this method has been generalized by [I2] in the whole space for the anharmonic operator.

We consider

$$
K(x, y)=\sum_{l} \varphi_{l}(y)\left\{\psi_{l}(x)-\varphi_{l}(x)\right\}
$$

and we have

$$
K(x, y)=\sum_{l} \psi_{l}(x)\left\{\varphi_{l}(y)-\psi_{l}(y)\right\}
$$

Remark : It is necessary to prove that $K(x, y)$ has a sense in the distributions space. For the first series the convergence comes from $[\mathrm{Sch}]$, and the second series represents $\delta(x-y)$ in $\mathcal{D}_{x}^{\prime}$ (see Appendix C). Indeed, we note that $K$ is an ultrahyperbolic operator. For, if

$$
\left\{\begin{array}{l}
V_{x}=-\triangle_{x}+q(|x|)+V(x), x \in \mathbb{R}^{2} \\
W_{y}=-\triangle_{y}+q(|y|)+W(y), y \in \mathbb{R}^{2}
\end{array}\right.
$$

then

$$
V_{x} K(x, y)=W_{y} K(x, y) \text { if } x \neq y
$$

Furthermore,

$$
K(x, y)=0\left\{\begin{array}{l}
\text { if }|x|>R \text { and } x \neq y \\
\text { or }, \\
\text { if }|y|>R \text { and } x \neq y
\end{array}\right.
$$

Indeed, if $x \neq y$ and since $\psi_{l}(x)=\varphi_{l}(x)$ if $|x|>R$,

$K(x, y)=\sum_{l} \psi_{l}(x) \varphi_{l}(y)=\sum_{l} \varphi_{l}(x) \varphi_{l}(y)=\delta(x-y)=0$ in $\mathcal{D}_{x}^{\prime}$.

In the same way we have, since $\psi_{l}(y)=\varphi_{l}(y)$ if $|y|>R$,

$K(x, y)=\sum_{l} \psi_{l}(x) \varphi_{l}(y)=\sum_{l} \psi_{l}(x) \psi_{l}(y)=\delta(x-y)=0$ in $\mathcal{D}_{x}^{\prime}$.

We can add that

$$
V_{x}^{m} K(x, y)=0 \text { if }|x|>R \text { and }|y|<R .
$$

We put for all $t>0$

$$
F_{t}(x, y)=\sum_{l \geq 1} e^{-t \lambda_{l}} \psi_{l}(x)\left\{\varphi_{l}(y)-\psi_{l}(y)\right\} .
$$

This series converges, because we can write

$$
F_{t}(x, y)=\sum_{l \geq 1} e^{-t \lambda_{l}} \psi_{l}(x) \varphi_{l}(y)-\sum_{l \geq 1} e^{-t \lambda_{l}} \psi_{l}(x) \psi_{l}(y)(\text { see }[\mathrm{Sch}])
$$

First, we prove that $F_{t}(x, y)=0$ if $|x|>R$ and $|y|<R$. We have 


$$
F_{t}(x, y)=\sum_{n=0}^{+\infty} \frac{t^{n}}{n !}\left(-V_{x}\right)^{n} K(x, y) .
$$

Indeed, $e^{-t \lambda_{l}}=\sum_{n=0}^{+\infty} \frac{t^{n}}{n !}\left(-\lambda_{l}\right)^{n}$, and by using (3.7), we obtain

$$
F_{t}(x, y)=\sum_{l \geq 1} \sum_{n=0}^{+\infty} \frac{t^{n}}{n !}\left(-\lambda_{l}\right)^{n} \psi_{l}(x)\left\{\varphi_{l}(y)-\psi_{l}(y)\right\}
$$

and then

$$
F_{t}(x, y)=\sum_{n=0}^{+\infty} \frac{t^{n}}{n !} \sum_{l \geq 1}\left(-\lambda_{l}\right)^{n} \psi_{l}(x)\left\{\varphi_{l}(y)-\psi_{l}(y)\right\}=\sum_{n=0}^{+\infty} \frac{t^{n}}{n !}\left(-V_{x}\right)^{n} K(x, y) .
$$

¿From (3.6) and (3.8) we can conclude.

Now, multiplying $F_{t}(x, y)$ by $e^{t \lambda_{1}}$, we obtain,

$$
e^{t \lambda_{1}} F_{t}(x, y)=0=\psi_{1}(x)\left\{\varphi_{1}(y)-\psi_{1}(y)\right\}+\sum_{l \geq 2} e^{-t\left(\lambda_{l}-\lambda_{1}\right)} \psi_{l}(x)\left\{\varphi_{l}(y)-\psi_{l}(y)\right\} .
$$

We prove (see Appendix D) that the limit, when $t$ tends to infinity, of the second term of the previous sum is equal to zero. Then we have $\psi_{1}(x)\left\{\varphi_{1}(y)-\psi_{1}(y)\right\}=0$ for $|x|>R$ and $|y|<R$. So, since $\psi_{1}(x)$ has no zero, (see [R.S]),

$$
\varphi_{1}(y)-\psi_{1}(y)=0 \text { for }|y|<R .
$$

Thus, (3.1) and (3.10) give

$$
\varphi_{1}(x)=\psi_{1}(x) \text { for } x \in \mathbb{R}^{2} .
$$

Now, we can write,

$$
[-\triangle+q(|x|)+V(x)]\left(\varphi_{1}(x)\right)=[-\triangle+q(|x|)+W(x)]\left(\varphi_{1}(x)\right),
$$

hence

$$
V(x)\left(\varphi_{1}(x)\right)=W(x)\left(\varphi_{1}(x)\right),
$$

and, since $\left(\varphi_{1}(x)\right)$ has no zero, we can conclude that

$$
V(x)=W(x) \text { for all } x \in \mathbb{R}^{2} .
$$

This uniqueness theorem for a compactly supported potential $V(x)$ concerns the Schrödinger operator $H=-\Delta+q(|x|)+V(x)$ associated with an increasing unbounded potential $q(|x|)$. Recall that when the potentials are bounded, authors use the scattering operator, the scattering amplitude or the Dirichlet to Neumann map to prove 
uniqueness results. Here, because of the unboundness potential $q$, the method is different and follows the method used by Isozaki [I2] for the anharmonic operator in $\mathbb{R}^{2}$. The uniqueness theorem is proved by using spectral data (the spectrum of $H$ being a pure point one).

We should note that potentials like $q(|x|)=|x|^{n},(n \geq 2)$ work fine if we replace the hypothesis $(h 2)$ by

$$
\left(h 2^{\prime}\right): \forall N \in \mathbb{N}^{*}, \exists C_{N} \in \mathbb{R}^{*+},|V(x)| \leq C_{N}(1+r)^{-N} e^{-\frac{2}{n+2} 2^{\frac{n+2}{4}} r^{\frac{n+2}{2}}} .
$$

Moreover, since the exponential function is increasing too rapidly $((h 4)$ does not hold ) and since the logarithmic function is not increasing sufficiently $((h 5)$ does not hold ), these two potentials do not satisfy our hypotheses.

\section{Acknowledgements}

The authors thank Yves Dermenjian, Hiroshi Isozaki and Peter Seibt for useful discussions. 


\section{References}

[Ab] A. Авbакhati-Mchachti, Systèmes semilinéaires d'équations de Schrödinger, Thèse numéro 1338, Université de Toulouse III, 1992, France.

[Ag1] S. Agmon, Lecture on Exponential Decay of Solutions of Second-order Elliptic Equations : Bounds on Eigenfunctions of $N$-body Schrödinger Operators, Mathematical Notes, Princeton University Press, New Jersey, 1982.

[Ag2] S. Agmon, Bounds on Exponential Decay of Eigenfunctions of Schrödinger Operators, Schrödinger Operators, (Como, 1984), Springer, Berlin, 1985, pp 1-38.

[E.E] D.E. Edmunds And W.D. Evans, Spectral Theory and Differential Operators, Oxford Mathematical Monograph, Clarendon Press Oxford University Press, New York, 1978.

[E.R] G. Eskin AND J. RALSTON, Inverse coefficient problems in perturbed half space, Inverse Problems 15, 1999 vol 3, p.683-699.

[G.R] J.C. Guillot and J. Ralston, Inverse Scattering at Fixed Energy for Layered Media, J. Math. Pures Appl. vol 1, 1999 p 27-48

[G.S.1] F. Gesztesy and B. Simon, Uniqueness Theorems in Inverse Spectral Theory for One-Dimensional Schrödinger Operators, Trans. Am. Math. Soc., 348, 1996, 349-373.

[G.S.2] F. Gesztesy and B. Simon, A New Approach to Inverse Spectral Theory, II. General Real Potentials and the Connection to the Spectral Measure, Ann. Math., 152 (2000), 593-643.

[I1] H. IsozAKI, Inverse scattering theory for wave equations in stratified media, J. Diff. Eq., vol 138, 1 (1997) p.19-54 .

[I2] H. Isozaki, Cours sur les Problèmes Inverses, Juin 1991, Université de Provence, Marseille, France.

[R.S] Reed and Simon, Method of Modern Mathematical Physics, vol. 4, Analysis of Operators, Academic Press, New York, (1978).

[Sch] L. Schwartz, Théorie des Distributions, Hermann \& Cie, Paris, 1966.

[Su] T. Suzuki, Ultra-hyperbolic Approach to some Multidimensional Inverse Problem, Proc. Japan Acad., 64, Ser. A, 1988.

[W] R. WedER, Multidimensional inverse problems in perturbed stratified media, J. Diff. Eq., vol 152, 1 (1999) p.191-239. 


\section{A Appendix}

We prove that the map $T$ is a contraction.

By $(h 5)$, we have:

$$
\exists C \in] 0,1\left[, \exists r_{0}, \forall r \geq r_{0},\left|\int_{r}^{+\infty} O\left(1 / q^{1 / 2} r^{2}, 1 / q^{3 / 2}, q^{\prime} / q^{2}\right)\right|<C .\right.
$$

Let

$$
\mathcal{F}=\left\{v=\left(v_{1}, v_{2}\right), \forall i, v_{i}:\left[r_{0},+\infty\left[\rightarrow \mathbb{R}, v_{1}=o\left(e^{-2 \int\left(q^{1 / 2}-\frac{\lambda}{\left.2 q^{1 / 2}\right)}\right.}\right), \sup _{r \geq r_{0}}\left|v_{2}(r)\right|<+\infty\right\}\right.\right.
$$

Note that $\left(\mathcal{F},\|\cdot\|_{\infty}\right)$ is a Banach space where

$$
\|v\|_{\infty}=\max \left(\left\|v_{1}\right\|_{\infty},\left\|v_{2}\right\|_{\infty}\right) .
$$

For $v \in \mathcal{F}$ we define $T(v)=w=\left(w_{1}, w_{2}\right)$ where

$$
w_{1}(r)=-\int_{r}^{+\infty} K_{1} v(t) d t \text { and } w_{2}(r)=\xi_{2}+\int_{r_{0}}^{r} K_{2} v(t) d t .
$$

By $(h 5)$ to $(h 7)$ we obtain that $w \in \mathcal{F}$.

We note now that $T$ is a contraction.

Indeed, if we define also for $v^{*} \in \mathcal{F}, T\left(v^{*}\right)=w^{*}=\left(w_{1}^{*}, w_{2}^{*}\right)$, we obtain :

$$
\begin{gathered}
\left\|w_{1}-w_{1}^{*}\right\|_{\infty} \leq \sup _{r \geq r_{0}} \int_{r}^{+\infty}\left|O\left(1 / q^{1 / 2} r^{2}, 1 / q^{3 / 2}, q^{\prime} / q^{2}\right)\left(v_{1}(t)-v_{1}^{*}(t)\right)\right| d t \\
+\sup _{r \geq r_{0}} \int_{r}^{+\infty} e^{-2 \int q^{1 / 2}-\frac{\lambda}{2 q^{1 / 2}}}\left|O\left(1 / q^{1 / 2} r^{2}, 1 / q^{3 / 2}, q^{\prime} / q^{2}\right)\left(v_{2}(t)-v_{2}^{*}(t)\right)\right| d t \\
\leq C\left\|v-v^{*}\right\|_{\infty} .
\end{gathered}
$$

We prove also easily that $\left\|w_{2}-w_{2}^{*}\right\|_{\infty} \leq C\left\|v-v^{*}\right\|_{\infty}$.

\section{B Appendix}

We prove that the constant $C_{\lambda, p}(V)$ exists.

Let $u_{1}$ and $u_{2}$ be a fundamental system of solutions for (2.19). Then there exists $c_{1}$ and $c_{2}$, constants such that:

$$
u_{p}(r)=c_{1} u_{1}(r)+c_{2} u_{2}(r)-u_{2}(r) \int_{1}^{r} u_{1}(t) f_{p}(t) d t+u_{1}(r) \int_{1}^{r} u_{2}(t) f_{p}(t) d t .
$$

By $(h 2)$ we obtain that

$$
\forall N \in \mathbb{N}^{*}, \exists C_{N}^{\prime} \in \mathbb{R}^{*+},\left|f_{p}(r)\right| \leq C_{N}^{\prime}(1+r)^{-N} e^{-\int q^{1 / 2}} .
$$


Therefore $u_{i} f_{p} \in L^{2}([1,+\infty[)$ for $i=1,2$.

Note that :

$$
\begin{gathered}
u_{p}(r)=\left(c_{1}+\int_{1}^{+\infty} u_{2}(t) f_{p}(t) d t\right) u_{1}(r)-\left(\int_{r}^{+\infty} u_{2}(t) f_{p}(t) d t\right) u_{1}(r) \\
+\left(c_{2}-\int_{1}^{+\infty} u_{1}(t) f_{p}(t) d t\right) u_{2}(r)+\left(\int_{r}^{+\infty} u_{1}(t) f_{p}(t) d t\right) u_{2}(r)
\end{gathered}
$$

Since $\int_{r}^{+\infty} u_{2}(t) f_{p}(t) d t=o(1)$ we deduce that $\left(\int_{r}^{+\infty} u_{2}(t) f_{p}(t) d t\right) u_{1}(r) \in L^{2}([1,+\infty[)$. By $(h 7)$ we obtain also that $\left(\int_{r}^{+\infty} u_{1}(t) f_{p}(t) d t\right) u_{2}(r) \in L^{2}([1,+\infty[)$.

Since $u_{2} \notin L^{2}\left(\left[1,+\infty[)\right.\right.$, we have that $c_{2}=\int_{1}^{+\infty} u_{1}(t) f_{p}(t) d t$.

Therefore we can prove that : $C_{\lambda, p}(V)=c_{1}+\int_{1}^{+\infty} u_{2}(t) f_{p}(t) d t$.

\section{Appendix}

Proof of $\sum_{p} \varphi_{p}(y) \varphi_{p}(x)=\delta(x-y)$ in $\mathcal{D}_{x}^{\prime}$.

We consider the function $\Phi_{N}(x, y)=\sum_{p=1}^{N} \varphi_{p}(y) \varphi_{p}(x)$ and $f$ an element of $\mathcal{C}_{c}^{\infty}$, then

$$
\begin{aligned}
<\Phi_{N}(\cdot, y), f(\cdot)> & =\int_{\mathbb{R}} \Phi_{N}(x, y) f(x) d x \\
& =\sum_{p=1}^{N} \varphi_{p}(y) \int_{\mathbb{R}} \varphi_{p}(x) f(x) d x \\
& =\sum_{p=1}^{N} \widetilde{f}_{p} \varphi_{p}(y),
\end{aligned}
$$

where $\widetilde{f}_{p}$ represents the coefficient of $f$ in the basis $\left\{\varphi_{p}\right\}_{p}$. Then,

$$
\begin{aligned}
\lim _{N \longrightarrow+\infty}<\Phi_{N}(\cdot, y), f(\cdot)> & =\lim _{N \longrightarrow+\infty} \sum_{p=1}^{N} \widetilde{f}_{p} \varphi_{p}(y) \\
& =\sum_{p=1}^{+\infty} \widetilde{f}_{p} \varphi_{p}(y) \\
& =f(y) \\
& =<\delta(\cdot-y), f(\cdot)>
\end{aligned}
$$

\section{Appendix}

We prove that the limit of the second term of (3.9) is equal to zero when $t$ tends to infinity.

We multiply the second term of (3.9) by a test function for fixed $N$ and fixed $x$, with $|x|>R$, then

$$
\left.<\sum_{n=2}^{N} e^{-t\left(\lambda_{n}-\lambda_{1}\right)} \psi_{n}(x)\left\{\varphi_{n}(y)-\psi_{n}(y)\right\}, f(y)\right)>=\sum_{n=2}^{N} e^{-t\left(\lambda_{n}-\lambda_{1}\right)} \psi_{n}(x)\left[\tilde{f}_{n}-f_{n}\right],
$$


where $\tilde{f}_{n}$ and $f_{n}$ represent the coefficients of $f$ relative to the basis $\left\{\varphi_{n}\right\}_{n}$ and $\left\{\psi_{n}\right\}_{n}$. We are going to study the following expression $\sum_{n=2}^{N} e^{-t\left(\lambda_{n}-\lambda_{1}\right)} \psi_{n}(x) f_{n}$. Recall that we are dealing with $|x|>R,|y|<R$, for all $N \in \mathbb{N}$, for all $f \in \mathcal{C}_{c}^{\infty}$ and for all real $t \in \mathbb{R}^{*+}$. So, we can write

$$
\begin{aligned}
\left\|\sum_{n=2}^{N} e^{-t\left(\lambda_{n}-\lambda_{1}\right)} \psi_{n}(x) f_{n}\right\| & =<\sum_{n=2}^{N} e^{-t\left(\lambda_{n}-\lambda_{1}\right)} \psi_{n}(x) f_{n}, \sum_{n=2}^{N} e^{-t\left(\lambda_{n}-\lambda_{1}\right)} \psi_{n}(x) f_{n}>^{\frac{1}{2}} \\
& =\left(\sum_{n=2}^{N} e^{-2 t\left(\lambda_{n}-\lambda_{1}\right)}\left|f_{n}\right|^{2}\right)^{\frac{1}{2}} \\
& \leq e^{-t\left(\lambda_{2}-\lambda_{1}\right)}\|f\|_{L^{2}} .
\end{aligned}
$$

So, for all $f \in \mathcal{C}_{c}^{\infty}$,

$$
\lim _{t \rightarrow+\infty}<\sum_{n=2}^{+\infty} e^{-t\left(\lambda_{n}-\lambda_{1}\right)} \psi_{n}(x)\left\{\varphi_{n}(y)-\psi_{n}(y)\right\}, f(y)>=0
$$

and this concludes the proof. 